Nloman 2015, 33(2), 95-105

Revista de Psicologia, Ciències de l'Educació i de l'Esport

ISSN: 1138-3194

Copyright ( 2015

www.revistaaloma.net

\title{
Acción socioeducativa en red con la infancia y adolescencia en situación de pobreza: análisis del programa CaixaProinfancia en Barcelona
}

\author{
Irene Cussó-Parcerisas, Jordi Longás-Mayayo \& Jordi Riera-Romaní \\ Universitat Ramon Llull
}

Recibido: 11-5-2015

Aceptado: 4-9-2015

Acción socioeducativa en red con la infancia y adolescencia en situación de pobreza: análisis del programa CaixaProinfancia en Barcelona

\begin{abstract}
Resumen. El artículo presenta como estudio de caso la aplicación del programa CaixaProinfancia en Barcelona impulsado por la Obra Social de la Fundación Bancaria Caixa d'Estalvis i Pensions de Barcelona, «la Caixa». Se presenta el marco teórico del programa y se analiza su incidencia y evolución en la ciudad. De los resultados de la investigación, cabe destacar las razones que han justificado el progresivo cambio hacia un planteamiento exigente de trabajo socioeducativo en red. A su vez, a partir de las nuevas experiencias, se presentan los aciertos, las expectativas y las limitaciones encontradas hasta la fecha en la implementación de este modelo emergente de acción socioeducativa. Los resultados apuntan hacia una acción más sostenible y eficaz de la atención integral de la infancia gracias al trabajo colaborativo en el territorio.
\end{abstract}

Palabras clave: pobreza infantil; acción socioeducativa; inclusión social; trabajo en red

The networked socio-educational response to childhood an adolescent poverty: an analysis of the CaixaProinfancia program in Barcelona

Summary. The article presents a case study of the CaixaProinfancia program implemented in Barcelona by «la Caixa» Foundation and includes an analysis of the program's effects and evolution in the city. Among the study's most noteworthy results is a look at the reasoning behind the program's gradual shift toward an approach demanding a networked socio-educational strategy. Then, based on these new experiences, there is a discussion of the successes, expectations and limitations encountered by the program to date in its implementation of this emerging socio-educational action model. The results suggest greater sustainability and effectiveness of integrated child services thanks to this cooperative work in the field.

Keywords: childhood poverty; socio-educational action; social inclusion; networked response

Correspondencia

Irene Cussó-Parcerisas

Grup de Recerca PSITIC

FPCEE Blanquerna (Universitat Ramon Llull)

c/ Císter, 34.08022 Barcelona

Email: irenecp2@blanquerna.url.edu 


\section{Introducción}

Un reciente análisis sobre los progresos de los objetivos marcados en la Estrategia Europa 2020 para las regiones europeas señala que siete regiones del sur de España se sitúan entre las diez últimas de la clasificación con relación a cumplir con los objetivos fijados en materia de empleo, investigación y desarrollo, educación, pobreza y exclusión social (Athanasoglou \& Dijkstra, 2014). En 2010, la Comisión Europea fijó como reto en la Estrategia Europa 2020 que el riesgo de pobreza se redujera a 20 millones de personas menos el año 2020 (Comisión Europea, 2010). Para medir los avances hacia ese objetivo se ha desarrollado un nuevo indicador comparativo a escala europea: People at risk of poverty or social exclusion (AROPE, por sus siglas en inglés).

El indicador AROPE define la población en riesgo de pobreza o exclusión social como aquella que sufre alguna de las siguientes situaciones: a) tener una renta personal equivalente inferior al 60\% de la media de la distribución de la renta (pobreza relativa o monetaria); b) estar en situación de carencia material severa (al menos carecen de 4 ítems de un listado de 9). Los elementos del listado son pagar el alquiler o letra; mantener el hogar a una temperatura adecuada; hacer frente a gastos imprevistos; comer carne, pollo o pescado (o equivalentes en dieta vegetariana) como mínimo tres veces por semana; pagar unas vacaciones de una semana/año; un coche; una lavadora; un aparato de televisión en color; un teléfono (fijo o móvil). Y c) o viven en hogares con baja intensidad de ocupación (hogares donde los miembros en edad de trabajar realizaron esta actividad en un 20\% menos respecto de su potencial de trabajo por el año de referencia (GonzálezBueno, Bello \& Arias, 2012).

El estado español alcanzó en 2013 una tasa AROPE del $27.3 \%$, por encima de la media de los 28 países (UE-28) de la Unión Europea, que fue del 24.5\%. La tasa AROPE para los menores de 16 años se sitúa también por encima de la UE-28, y alcanza el 31.9\% y $27.3 \%$, respectivamente. Cabe destacar que son los jóvenes de entre 16-24 años los más afectados por el fenómeno, con una tasa de un 36.9\% (Eurostat, 2015). Este indicador pone de relieve la problemática de la pobreza y exclusión social en general en nuestro país y, en particular, sobre el colectivo de menores y jóvenes adultos. Al mismo tiempo, el indicador apuesta por una nueva definición de los contextos de pobreza. Se amplían las dimensiones que hay que tener en cuenta para analizar el fenómeno, no solo se trata de una cuestión económica (de ingresos), sino que identifica otros elementos que caracterizan estas situaciones.

La tasa AROPE para los menores de 16 años en España en el período 2005-2013 ha aumentado progresivamente, situándose siempre por encima de la UE-27 (Eurostat, 2015) y poniendo de manifiesto que la pobreza es un problema estructural que se ha agravado con la última crisis económica (González-Bueno et al., 2012).
En nuestro contexto, diferentes factores contribuyen a la vulnerabilidad social de la infancia y la juventud: la participación en el mercado de trabajo de forma precaria y discontinua o el paro de larga duración, la dificultad de acceso a la vivienda, las transformaciones en la institución familiar, y también unas políticas familiares y sociales con escaso impacto sobre la pobreza. Por ejemplo, en 2011 España dedicó un 0.5\% del PIB a ayudas monetarias a familias con niños, respecto al $1.4 \%$ de media de la Unión Europea. Además, no contamos con un sistema universal de prestaciones por hijos a cargo, uno de los mecanismos probados como efectivos en otros países, aunque no exclusivo, para la reducción de la pobreza en este colectivo (González-Bueno \& Bello, 2014:38). Todos estos elementos inciden en la infancia y el incremento de la pobreza, que se define también como una pobreza familiar, transmitida generacionalmente y asociada a la estructura social y a los ejes de desigualdad de género, clase y etnia.

A partir del indicador AROPE, las cifras de 2013 ponen de relieve la intensificación del fenómeno de la pobreza y exclusión según variables de estructura social. Según el tipo de hogar, los que presentan una mayor tasa AROPE en España son los hogares con un adulto y niños dependientes (hogares con menores de 18 años o adultos de entre 18 y 24 inactivos), un 47.6\%; en relación con la nacionalidad, las personas extranjeras no europeas registran una tasa AROPE del 60.3\%. Finalmente, según el nivel de educación alcanzado, las personas con educación primaria o inferior (incluye analfabetos) o personas con educación secundaria de primera etapa son las que registran una tasa AROPE más elevada $32.5 \%$ o $35.1 \%$, respectivamente, mientras que la tasa disminuye a mayor nivel de estudios, que es del $13.3 \%$ para las personas con estudios superiores (INE, 2014a, 2014b, 2014c).

Según datos del Departamento de Estadística del Ayuntamiento de Barcelona, basados en la última edición de la Enquesta de Condicions de Vida i Hàbits de la Població de Catalunya (ECVHP) realizada por el Institut d'Estadística de Catalunya (Idescat) y el Institut d'Estudis Regionals i Metropolitans de Barcelona (IERMB), en el año 2011, un 18.3\% de personas en la ciudad vivía bajo el umbral de pobreza establecido, como tener una renta personal equivalente inferior al $60 \%$ de la media de la distribución de la renta. Entre los menores de 16 años, este porcentaje es del 20.4\%. La tasa es inferior a la cifra estimada para la población en su conjunto y para los menores de edad en Cataluña, que asciende a $21.9 \%$ y $28 \%$, respectivamente.

Según la misma fuente, habría un $28.1 \%$ de personas en riesgo de pobreza o exclusión social (AROPE) en Barcelona. Para el colectivo de menores de 16 años en la ciudad la tasa AROPE se situaba en el $29.2 \%$ y el $36.1 \%$, según el umbral de pobreza, Cataluña y Barcelona ciudad, respectivamente (Idescat, 2014). Lo que significa que, cuando comprendemos el fenómeno desde una perspectiva más compleja y en más dimensiones, incluyendo medidas de privación material y de 
participación en el mercado de trabajo, la situación de la ciudad también alcanza cifras preocupantes, lejos de los objetivos de la Estrategia Europa 2020. (Departament d'Estadística de Barcelona, 2012a y 2012b).

Respecto a la supuesta transmisión intergeneracional de la pobreza a la que nos hemos referido, los datos proporcionados por el INE a través de un módulo especial de la Encuesta de Condiciones de Vida 2012 dedicado a esta temática son reveladores. En ellos se pone de relieve que los adultos tienen una mayor tasa de pobreza relativa cuando tienen padres con menor nivel de estudios y también en el caso de haber experimentado dificultades económicas en el hogar de origen en su adolescencia (INE, 2012). De ahí también la preocupación por los elevados porcentajes de fracaso escolar en nuestro país entendido como la no graduación de los estudios obligatorios (Marchesi \& Hernández-Gil, 2003; Calero, Chois \& Waisgrais, 2010). En el curso 2010-2011, solo un 76.9\% consiguió el graduado en Educación Secundaria Obligatoria. Pero, al mismo tiempo, sabemos que muchos de ellos, aun habiendo obtenido el graduado, no siguen estudiando. Concretamente, en 2013, un 23.5\% de jóvenes de 18 a 24 años abandonaron prematuramente los estudios (GonzálezBueno \& Bello, 2014:46). Sin embargo, los datos del informe PISA 2012 confirman que en la distribución de este fracaso escolar no es ajeno el origen de los estudiantes y las condiciones socioeconómicas y culturales del alumnado y sus familias, lo cual influye de forma más acusada en los resultados y retrasa la posición de España en relación con el promedio de la OCDE (MECD, 2013).

Diversas investigaciones en nuestro país señalan la correlación entre fracaso escolar y vulnerabilidad social (Sarasa y Sales, 2009; Gil-Flores, 2011) o entre la asociación del fracaso escolar con la marginalidad y la exclusión social (Boada et al., 2011). Se constata que las personas con un menor nivel de cualificación (analfabetos o educación primaria) tienen una tasa de paro alrededor del $54.1 \%$ y $42.5 \%$, respectivamente (primer trimestre de 2015), tasa que se reduce a mayor nivel de estudios (INE, 2015). Si consideramos la relación entre nivel de educación alcanzado y bienestar posterior, la educación se postula como uno de los mecanismos fundamentales para abrir oportunidades a la infancia en el presente y como futuros adultos, una de las pocas claves para romper el círculo vicioso de la pobreza que parece condenar a los hijos de las familias con menos recursos a heredar los mismos mecanismos de exclusión social (Riera, 2012; Garcia Alegre, 2013).

Constatamos que intervienen diferentes variables en las situaciones de pobreza y exclusión social. Por lo tanto, se hace necesario adoptar, para la comprensión del fenómeno, un pensamiento complejo que nos permita elaborar una aproximación desde una lógica sistémica, tal como nos propone Morin (2005). Este cambio en la definición de la realidad implica también un cambio de paradigma en la forma de dar respuesta a estas problemáticas y necesidades educativas y sociales. En este sentido, Civís y Riera (2007) proponen el desarrollo de una nueva pedagogía social y comunitaria que debe pasar de la acción asistencial y fragmentada a la acción sociopedagógica y en red para enfrentarse a los retos educativos actuales desde el ámbito formal, no formal e informal. Siguiendo a los mismos autores, se apela a la corresponsabilidad social entendiendo el hecho educativo como un todo no delegable a un solo agente, sino que compromete a toda la sociedad. Para ello se requieren estrategias de acción integral conjunta, coordinada, corresponsable, eficiente y sostenible en un territorio determinado llevada a cabo en red por los agentes con responsabilidades socioeducativas (escuelas, servicios sociales, servicios culturales, de salud, etc.) (Civís \& Riera, 2007; Riera \& Cussó, 2013; Ballester, 2014).

Constatamos un avance en esta línea en las dos últimas décadas con el desarrollo de programas de acción socioeducativa transversales y bien coordinados, a escala internacional y nacional, como las Zones d'action Prioritaire (Francia, 1982), las Education Action Zones (Reino Unido, 1998), las Extended Schools (Reino Unido, 2004), The Harlem Children's Zone (Estados Unidos, 1990) o Interxarxes y Redes Educativas Locales (España, 2000), (Díaz-Gibson, Civís y Longás, 2013).

Definimos las redes locales de acción socioeducativa como «redes organizacionales de carácter formal, integradas por actores (instituciones y entidades públicas y privadas), orientadas hacia el logro de metas socioeducativas comunes, que basan su acción en la colaboración a través de una programación estratégica conjunta y que son promotoras de capital social en la comunidad» (Díaz-Gibson et al., 2013:216).

En este sentido, las redes socioeducativas se regirán por criterios de proximidad (respuesta a las necesidades del territorio), transversalidad (visión integral e integradora de la acción), horizontalidad (organización y estructura de relaciones que no es jerárquica), corresponsabilidad (existencia de un compromiso compartido), colaboración (reconocimiento e interdependencia en las relaciones) y, finalmente, proactividad y proyección (planificación estratégica y orientación a logros), principios desarrollados en diferentes investigaciones de Longás y Civís (2010), Díaz-Gibson, Civís, Longás y López, (2010), Díaz-Gibson y Civís (2011), y Civís y Longás (2015).

La Obra Social de «la Caixa» inició en 2007 el programa CaixaProinfancia (CPI) con la finalidad de ampliar las oportunidades educativas de la infancia -entre 0-16 años- que se encuentra en situación de vulnerabilidad a causa de la pobreza. Al inicio de la crisis económica se diseñó el programa alineado en las directrices de los programas marco de la Unión Europea contra la exclusión social mediante el fomento de políticas de mejora de la equidad, la cohesión social y la igualdad de oportunidades.

En el período 2007-2013, el programa atendió en toda España a 222.167 menores y a 139.664 familias, en las 11 áreas metropolitanas españolas más pobladas con mayores índices de pobreza infantil: Baleares, Barcelona y su área metropolitana, Bilbao, Madrid y su 
área metropolitana, Málaga, Murcia, Sevilla, Las Palmas de Gran Canaria, Tenerife, Valencia y Zaragoza. En todo el proceso construyó un tejido de atención social formado por más de 350 entidades sociales y diez administraciones públicas. Especialmente, el programa focaliza su acción en el apoyo social y educativo mediante un trabajo sociofamiliar e interdimensional.

Dada la singularidad e importancia de esta iniciativa de colaboración público-privada en la atención a la infancia, nos planteamos como objetivo de esta investigación analizar las claves teórico-prácticas que sustentan su implementación y han guiado su evolución hasta el pilotaje del trabajo en red en algunos barrios de la ciudad de Barcelona.

\section{Método}

En este estudio nos planteamos conocer cómo se está desarrollando la evolución de CPI hacia formas de acción socioeducativa en red en la ciudad de Barcelona. Seguimos la metodología de estudio de caso único de carácter exploratorio (Coller, 2000), entendiendo que es la opción más adecuada para comprender los aciertos y las dificultades de esta experiencia pionera que se encuentra en plena fase de desarrollo. Se justifica por la necesidad de transferir los aprendizajes que se generen en esta experiencia a las otras experiencias piloto que, con algo más de retraso, se han iniciado en otras ciudades españolas.

Ante la dificultad de estudiar un programa en pleno proceso de implementación se ha optado por presentar una narrativa cronológica, ordenada en cuatro etapas: a) inicio del programa y claves que definen su primer desarrollo; b) análisis de la incidencia del programa CPI en la provincia y ciudad de Barcelona antes de iniciar el proceso de cambio; c) análisis de las bases del nuevo modelo de redes socioeducativas territoriales que definen la reorientación del programa, y d) primera valoración del pilotaje de redes socioeducativas.

Para conocer las bases del programa hemos recurrido al análisis documental del documento marco Programa CaixaProinfancia: modelo de promoción y desarrollo integral de la infancia en situación de pobreza y vulnerabilidad social (Grupo de investigación PSITIC, 2013a). Para el estudio de la incidencia del programa en Barcelona se utiliza el estudio del diagnóstico de necesidades de atención y recursos CPI CaixaProinfancia. Informe estudio del mapeo. 2012. Zona de Barcelona elaborado por Boadas \& Ricciardelli (2012). El análisis de las claves del nuevo modelo de trabajo en red que se está pilotando se ha realizado a partir de las entrevistas a un técnico y a un asesor del programa y del material de uso interno facilitado que lo describen.

Finalmente, para la valoración de la implementación de los pilotajes en Barcelona se constituyó, en noviembre de 2014, un grupo de discusión con los agentes representantes de las experiencias piloto en la ciudad que contó con los siguientes participantes: técnico del programa CaixaProinfancia de la Obra Social «la Caixa»; asesora del programa CPI de Barce- lona; trabajadora social y coordinadora técnica del pilotaje de Verneda-La Pau (Sant Martí); educadora social y coordinadora técnica del pilotaje en Zona Nord Nou Barris y técnico del Plan Comunitario de Ciutat Meridiana y participante en la mesa del pilotaje de Zona Nord de Nou Barris. A partir de la perspectiva propia de los participantes se realizó un análisis DAFO (fortalezas y debilidades internas, amenazas y oportunidades del contexto) en relación con el programa CPI y de las experiencias piloto iniciadas. Se ha analizado la transcripción de la grabación del grupo de discusión teniendo como referencia los principios del trabajo en red que hemos presentado.

\section{Resultados}

\section{Claves del desarrollo y evolución del programa CaixaProinfancia}

El programa CPI se inicia en la segunda mitad del año 2007. Se concibe como una cartera de bienes y servicios, principalmente de tipo educativo y apoyo a la familia, que administran entidades del tercer sector agrupadas en redes. Tras un despliegue territorial en las 11 áreas más pobladas de España, muy centrado en la administración de la cartera y su extensión máxima dentro de los límites fijados por un presupuesto anual de 50 millones de euros, se observan, por parte del equipo gestor, algunas dificultades que llevan a abrir un período de reflexión. Es indudable que el programa se caracteriza por su dinamismo y flexibilidad, con el que se busca el aprendizaje continuo; de modo que, a pesar del corto período de implementación, no se duda en introducir aquellos cambios con voluntad de mejora considerados necesarios.

Tras la implementación en el período 2007-2010 se detecta la necesidad de fortalecer el modelo de acción. Por este motivo se encarga al grupo de investigación PSITIC de la Universidad Ramon Llull el liderazgo de un proceso de reflexión participativo con las 31 entidades que coordinan las redes de trabajo en cada ciudad y que se extiende entre los años 2010-2012. El intercambio de experiencias y reflexiones permite identificar las principales fortalezas y debilidades del CPI y se acuerdan las bases para desarrollar un modelo de acción socioeducativa más innovador y focalizado, preferentemente, en aquellos lugares cuya necesidad sea mayor y la viabilidad lo justifique.

El trabajo se orientó a construir, conjuntamente con las entidades coordinadoras de las redes locales, los expertos de las universidades y del trabajo social, y el equipo técnico de CPI, aquellas mejoras en la acción socioeducativa del programa que se estimaban convenientes y posibles. El proceso culminó con la redacción del modelo general Programa CaixaProinfancia: modelo de promoción y desarrollo integral de la infancia en situación de pobreza y vulnerabilidad (Grupo de investigación PSITIC, 2011). A partir del curso 2011-2012, las entidades implementaron los primeros cambios que exigía la revisión del modelo y se trabajó participativamente 
para editar el Modelo de Acción Social, eje fundamental sobre el que gira toda la acción socioeducativa con la población destinataria del programa, y las guías del subprograma de Refuerzo Educativo y del subprograma de Atención Psicoterapéutica. Estos documentos fueron publicados en el curso 2012-2013 (Grupo de investigación PSITIC, 2013b, 2013c, 2013d).

El modelo de acción socioeducativa generado en este período se refleja conceptualmente en la Figura 1. Se puede observar cómo a partir de un proceso sistemático de acción/acompañamiento social se desarrollan varios procesos complementarios de apoyo (planes de acción social multidimensional) personalizados para cada menor y familia.

Propiamente, la intervención con las familias se realiza mediante la combinación de las siguientes estrategias-acciones generales, reforzadas por la prestación gratuita de bienes cuando son necesarios:

- Apoyo a la familia: acompañamiento profesional, social, psicológico y pedagógico, de responsabilidad y desarrollo.

- Programas de inclusión educativa: refuerzo educativo y apoyo a la escolarización de los menores, educación en el tiempo libre, apoyo psicológico personalizado, y promoción de su salud integral.

- Fortalecimiento de la «red social»: promoción de la relación con el entorno y entre iguales, y coordinación en red con los agentes socioeducativos (servicios sociales, escuelas, entidades, etc.) para asegurar una acción integral y coordinada centrada en el niño o la niña (en red), que amplíe el «capital social» de dichos menores y familias.
La interacción e interdependencia de los ámbitos planteados es indiscutible, como también que las acciones posibles propuestas para cada ámbito puede ser enriquecida de forma significativa en el futuro. Este planteamiento «matricial» de la intervención permite crecer de forma sistémica con nuevos ámbitos de actuación, tan necesarios como la promoción de la ocupación o la participación ciudadana.

A partir del año 2012 y hasta el momento actual, los ejes principales de la mejora y el desarrollo del programa son a) la firma de acuerdos con los principales ayuntamientos en los que incide el programa para ajustar la colaboración, b) la continuación de la reconceptualización del modelo concretando las guías de procesos del resto de subprogramas que se vienen desarrollando, c) la sistematización de la evaluación, d) la formación continuada de los agentes socioeducativos, y e) el pilotaje de experiencias de creación de redes socioeducativas a escala local en favor de la infancia vulnerable a partir de un mapeo detallado de necesidades y recursos por ciudad/territorio elaborado en el curso 2011-2012.

\section{Incidencia del programa CPI en la provincia y ciudad de Barcelona}

CPI en la provincia de Barcelona se desarrolla en más de 50 municipios a través de cinco entidades del tercer sector que implementan el programa, ya sea a través de ellas mismas o de las entidades colaboradoras con las que cuenta cada una. De este modo, constituidas en red actúan en el territorio para acompañar social-

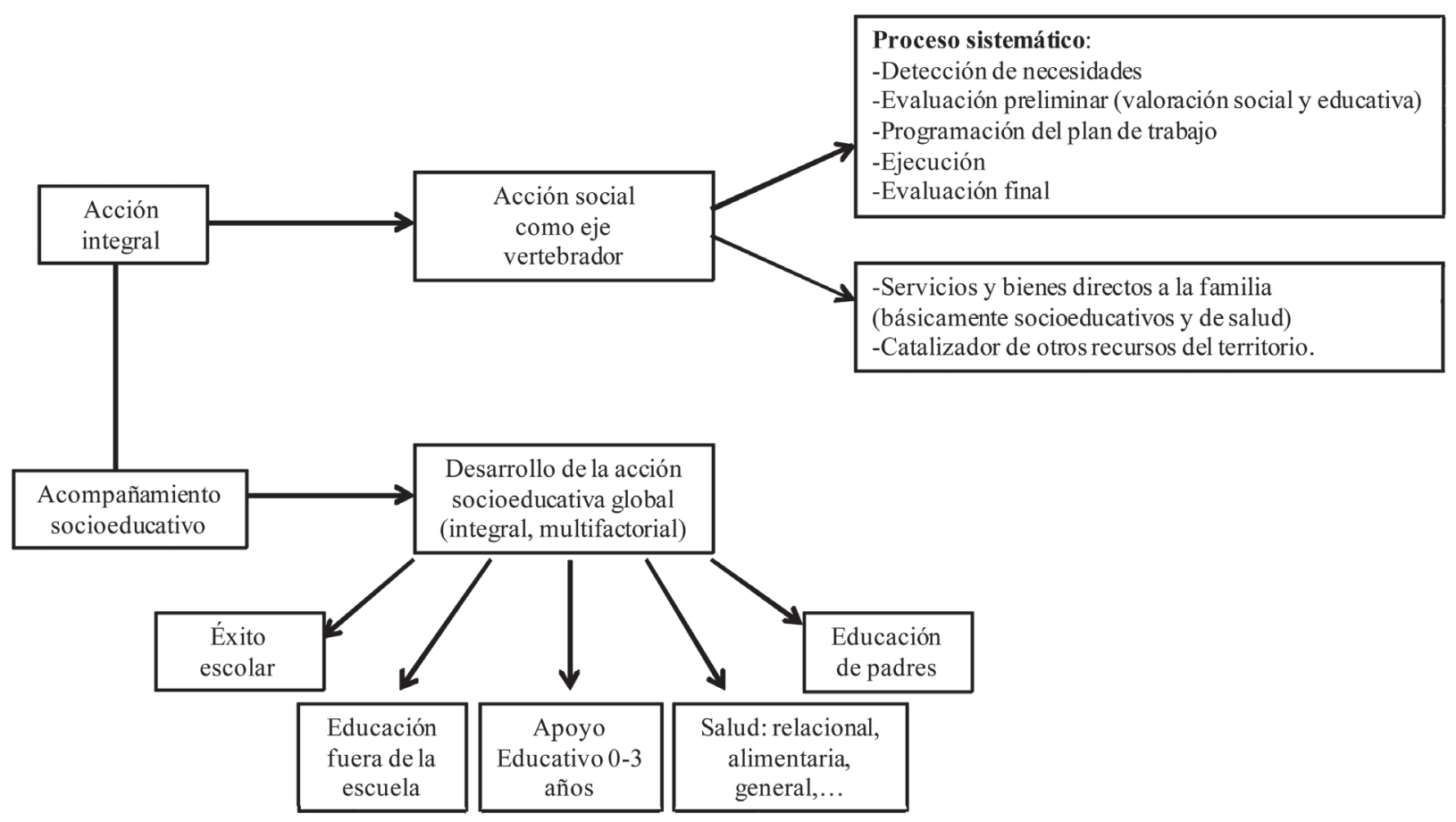

Figura 1. Modelo de acción socioeducativa del programa CaixaProinfancia.

Fuente: Elaboración propia basada en el modelo general publicado en Programa CaixaProinfancia: Modelo de promoción y desarrollo integral de la infancia en situación de pobreza y vulnerabilidad social (2011), reeditado en 2013. 
mente a los menores y a las familias en situación de pobreza y vulnerabilidad. Las entidades coordinadoras en la provincia de Barcelona son Cáritas Diocesana de Barcelona; Associació Professional Serveis Educatius de Catalunya (APSEC); Federación y Atención a la Infancia y la adolescencia (FEDAIA); Casal dels Infants per a l'Acció Social als Barris (Casal d'Infants del Raval) y Save the Children.

Las cinco redes coordinadoras de CPI se distribuyen por toda la ciudad, de manera que encontramos entidades que implementan el programa en todos los distritos. Algunas conclusiones que podemos avanzar del análisis del mapeo llevado a cabo en el curso 20112012 han permitido identificar la distribución del programa en la ciudad y la concordancia con las necesidades del territorio (Boadas \& Ricciardelli, 2012).

En 2012, el programa atendió en la ciudad de Barcelona a 7.087 menores y 4.887 familias, lo que representa un 3\% de la población infantil (0-16 años) del municipio (223.848), según Idescat (2012). Teniendo en cuenta que el $20.4 \%$ de los menores de 16 años en 2011 en la ciudad se sitúa en niveles de pobreza relativa podemos concluir que el programa incide sobre el $15 \%$ de este colectivo.

Las ayudas de CPI en la ciudad en 2012 se distribuyeron de la siguiente manera: a) $50.66 \%$ del total de menores tuvieron servicios socioeducativos (30\% solo servicios y $20.3 \%$ servicios y bienes) y b) $49.3 \%$ del total de menores tuvieron ayudas de bienes. La evolución de la distribución de bienes respecto a años anteriores muestra la tendencia hacia una acción más integral.

Según el tipo de ayuda recibido, el refuerzo educativo es el servicio con más participantes (4.625 menores), seguido de las actividades de educación no formal y tiempo libre (2.419 menores) y los recursos para la promoción de la salud (1.732 menores). La atención o terapia psicosocial suma 829 participantes y el apoyo familiar iniciado en el curso 5 (2011-2012), 126 padres y madres (2012).

La constatación, a partir de los mapeos realizados en todo el territorio, de la extensión del programa en volumen de beneficiarios, la diversidad de servicios y entidades, y la voluntad de seguir avanzando para la mejora de la calidad de la atención socioeducativa integral de la infancia, la optimización de recursos, la búsqueda de eficiencia y sostenibilidad del programa, conducen al desarrollo de nuevas formas de trabajo en red que actualmente se conciben como modelos más óptimos en la atención socioeducativa de la infancia.

De común acuerdo con la Administración local y las entidades se han iniciado experiencias destinadas a pilotar el desarrollo de redes territoriales (de distrito o barrio) para mejorar la atención y el apoyo socioeducativo a la infancia vulnerable. En ellas se propone el trabajo coordinado de los diferentes agentes socioeducativos (servicios sociales, escuelas, entidades, etc.) para optimizar: a) los sistemas de detección, derivación y colaboración en el acompañamiento de familias y menores, flujos, y b) la gobernanza de los recursos socioeducativos del territorio y su diseño ajustado a las necesidades reales.

\section{Promoción de redes socioeducativas territoriales de atención a la infancia y adolescencia en situación de vulnerabilidad social}

En el año 2013 se estima que la forma más eficiente y a la vez innovadora de atender a la infancia es mediante el desarrollo de estrategias de concertación entre los ditintos actores socioeducativos que tienen incidencia sobre la infancia vulnerable de una misma zona. Se estima necesario promover mesas de infancia para articular el trabajo en red dentro de un mismo territorio. Se busca con ello activar la colaboración entre entidades, servicios sociales, centros educativos, etcétera, en pos de una acción más integral y sostenible. Antes de extender la propuesta a todos los territorios en los que está presente CPI se decide pilotar el desarrollo de 30 experiencias diseminadas en las 11 ciudades o áreas metropolitanas de referencia. En función de los resultados se extenderá a todo el programa el modelo con la correspondiente transferencia de los aprendizajes realizados.

La construcción de una red local de acción socioeducativa es un proceso relativamente largo y complejo, que precisa de la realización simultánea de diversas acciones orientadas a conocer mejor los recursos y las oportunidades del territorio, organizar los flujos, así como activar las relaciones y sinergias entre los agentes del territorio y coordinar las acciones que desarrollan siguiendo los principios de proximidad, transversalidad, horizontalidad, colaboración, corresponsabilidad y proactividad y proyección descritos anteriormente.

En el caso de CPI, y con el fin de garantizar el acompañamiento socioeducativo de calidad a la infancia y adolescencia en situación de vulnerabilidad a causa de la pobreza, se focaliza la atención en incrementar las oportunidades educativas y de inclusión social de la infancia. Para promover y construir las redes se propone, en primer lugar, llegar a un acuerdo político y técnico que legitime el proceso y, posteriormente, desarrollar cuatro pasos básicos ilustrados en la Figura 2.

- Constitución de un equipo promotor. Debería estar integrado por servicios sociales de la zona, la entidad coordinadora «socia» de CPI (la actual o de nueva incorporación según el territorio) y técnicos del programa CPI (del equipo de gestión y asesores según el caso y momento). Es totalmente aconsejable contar también, desde el inicio, con los centros educativos. A tal fin será de gran ayuda el establecimiento de acuerdos marco de colaboración con los ayuntamientos y gobiernos autonómicos que corresponda.

- Mapeo del territorio y propuesta de constitución de mesa de infancia. Se pretende conocer y reconocer los recursos y servicios que se desarrollan en la zona de actuación, las principales entidades que lo llevan a cabo y los déficits de la acción social en el ámbito de actuación. A partir de este diagnóstico de análisis de necesidades y de entidades, será posible 
priorizar líneas de acción y desarrollar estrategias y planes de actuación a escala de la red local.

- Constitución de la mesa de infancia. Se debería invitar a los agentes que compongan la mesa a una primera reunión constitutiva. La estrategia que hay que seguir pretende asegurar el éxito, por lo que es aconsejable, antes de proceder a realizar convocatorias formales, prever el sistema de comunicación y acercamiento más efectivo. En muchos casos puede ser recomendable aprovechar estructuras de participación técnico-profesionales que ya estén establecidas en el territorio (planes comunitarios, mesas educativas, etc.). Hay que señalar la conveniencia de generar un escenario nuevo no contaminado por dinámicas relacionales previas, que sea percibido por los participantes como un espacio real de innovación colaborativa.

- Elaboración e implementación del plan de territorio. Una vez constituidas la mesa de infancia y la comisión gestora (que puede ser la prolongación o ampliación del equipo promotor de la red), deberá elaborarse un plan estratégico del territorio, con objetivos de mejora en la atención de la infancia y adolescencia a medio-largo plazo y un plan de acción para el curso inmediato. La secuencia del «ciclo de gestión» (necesidades, objetivos, recursos, acciones y evaluación) debe guiar también el trabajo en red.

\section{Valoración del pilotaje de las redes socioeducativas territoriales en Barcelona}

En 2013 se impulsaron en Barcelona tres experiencias piloto de red local de acción socioeducativa, en Nou
Barris (barrio Zona Nord Nou Barris), en Sant Martí (barrio Verneda-La Pau) y otra en Ciutat Vella (barrio Gótico). El desarrollo de las experiencias no es homogéneo, aunque en estos momentos ya se han completado los tres primeros pasos más "procesuales»y constitutivos y se trabaja en el cuarto en las dos primeras experiencias mencionadas. El propósito es promover en estos territorios la coordinación entre los agentes socioeducativos locales (escuelas, servicios sociales, entidades) y el establecimiento de un plan de acción para estas zonas, así como protocolos e instrumentos comunes. La creación de estas redes proporciona la oportunidad de crear estructuras y dinámicas nuevas en territorios socialmente vulnerables con la finalidad de aprovechar eficientemente los recursos de un territorio, y dar respuesta desde una intervención más compleja de acuerdo con las nuevas dinámicas de pobreza y exclusión social que afectan a la infancia en nuestro contexto.

A partir de las valoraciones y reflexiones aportadas por los representantes de los agentes que participan en dos de las experiencias piloto en Barcelona más avanzadas identificamos hasta qué punto los pilotajes impulsados están desarrollando los principios que orientan el trabajo socioeducativo en red mencionados anteriormente y qué elementos aparecen como retos en la puesta en marcha de estos procesos hasta la fecha.

En relación con la proximidad, las experiencias piloto promovidas desde CPI focalizan su acción en la infancia vulnerable en un territorio determinado. Se han empezado los pilotajes en áreas identificadas con altas necesidades donde el programa no tenía presencia inicialmente. Se busca la contextualización de las ac-

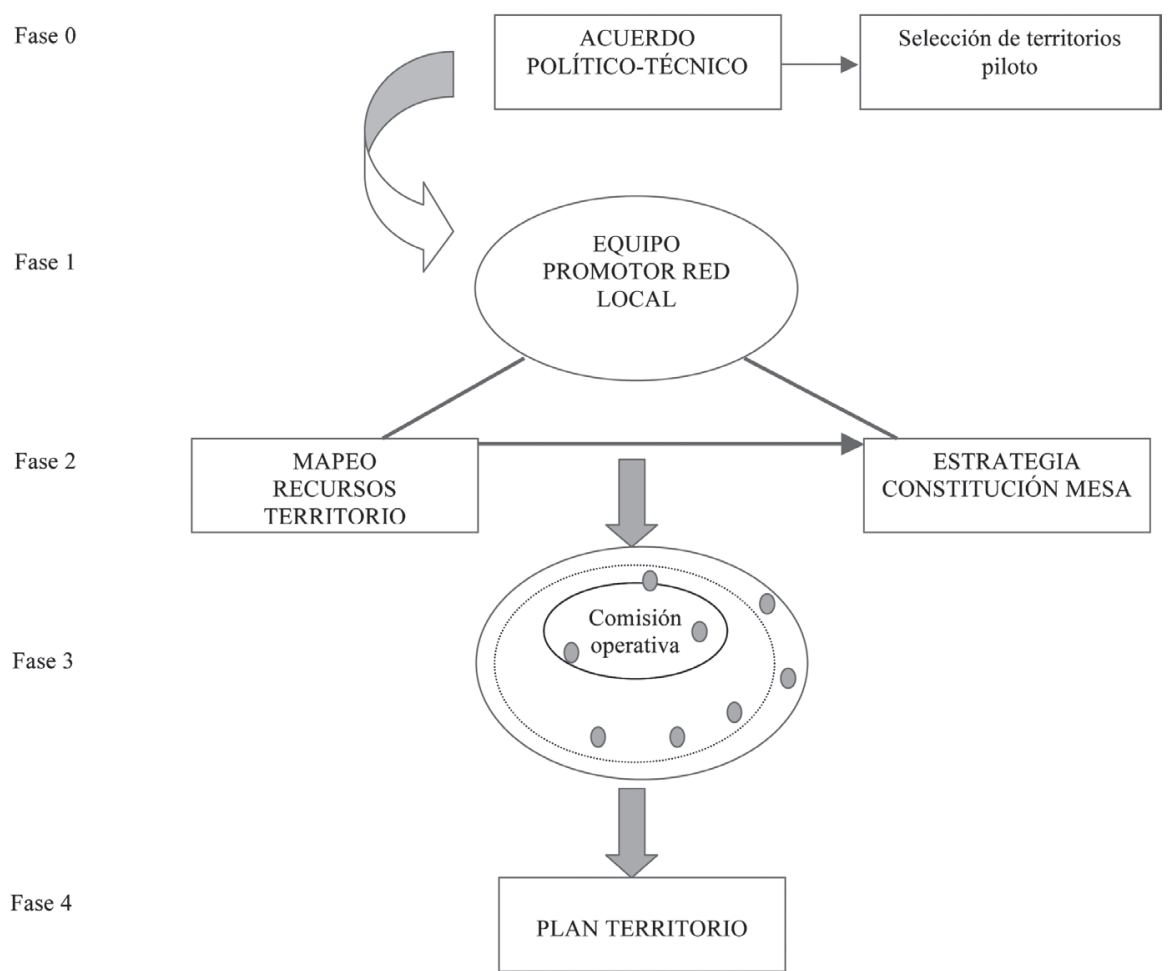

Figura 2. Proceso para la promoción de una red local de acción socioeducativa Fuente: Elaboración propia. 
ciones en una determinada zona realizadas a través de entidades y proyectos existentes en el territorio, en el cual CPI se inserta de forma complementaria como un agente más, adaptándose a las necesidades del entorno. Los actores entrevistados reconocen que la creación de redes territoriales supone un cambio de enfoque importante en el programa, ya que mayoritariamente en un inicio fue configurado a partir de redes creadas bajo principios de afinidad entre entidades. Esta nueva práctica constituye un reto, al mismo tiempo que genera nuevas formas de hacer e influye en el resto del programa avanzando hacia criterios de territorialización.

Las experiencias piloto ponen el foco de su acción en atender de forma integral las necesidades de los niños y adolescentes y sus familias, siguiendo los principios del modelo socioeducativo de acción propuesto desde CPI (Grupo de investigación PSITIC, 2011, 2013a). Ello significa que los diferentes agentes desarrollan una acción interprofesional que requiere de un enfoque interdisciplinar para responder, en nuestro caso, a la situación compleja de vulnerabilidad social de las familias. Cada profesional aporta desde su especialidad la información necesaria para comprender las diferentes dimensiones del caso, y construir un marco único de acción a partir de conocimientos y criterios compartidos (Riera, 1997; Laguna \& Riera, 1998). Por ejemplo, en los pilotajes de la ciudad de Barcelona forman parte de las mesas de trabajo directores y educadores de escuelas primarias y secundarias, trabajadores sociales de los servicios sociales, pediatras, psicólogos, educadores sociales o técnicos comunitarios. Sin embargo, los mismos agentes manifiestan que queda pendiente incorporar también a otros agentes del ámbito laboral y de la vivienda, ya que son aspectos fundamentales para la inclusión social de las familias.

Sin duda, la acción transversal solventa un déficit inicial de CPI donde la administración no colaboraba. La inclusión de las instituciones públicas es un esfuerzo de colaboración que el programa viene realizando desde 2010. Al mismo tiempo, la transversalidad constituye un reto también en la práctica diaria de los profesionales, ya que supone superar la lógica asistencialista de gestión de recursos y avanzar hacia una cultura de trabajo colaborativo en la que no solo se trata de prestar o distribuir los recursos y servicios disponibles, sino también de acompañar a las familias en un proceso de transformación social. En definitiva, podemos decir que la voluntad de avanzar hacia la transversalidad de las acciones también conlleva desarrollar inherentemente el principio de colaboración, fundamental en las redes socioeducativas. La cultura colaborativa ya es un hecho visible en los pilotajes en marcha en Barcelona, aunque los entrevistados ponen de relieve que hace falta consolidarla, ya que a menudo las diferencias institucionales frenan su desarrollo.

La participación de los diferentes agentes en las mesas de trabajo de los pilotajes se realiza desde un criterio de horizontalidad. Es decir, no existe una relación jerárquica entre los agentes. Así, pues, en la mesa interactúan profesionales de distintas instituciones que tienen un interés común: atender a la infancia y a sus familias en un territorio determinado. El pilotaje de la Zona Nord de Nou Barris, el cual incluye los barrios de Torre Baró, Vallbona y Ciutat Meridiana, se estructura a partir de representantes de comisiones ya existentes en la zona, por ejemplo, la comisión de infancia, de juventud, de educación, de inserción socio-laboral, la de atención comunitaria, la de atención de salud, la de atención psicoterapéutica y el plan comunitario. En cambio, el pilotaje en el barrio de Verneda-La Pau, al no contar con estructuras existentes que aglutinan los agentes de la zona, se ha constituido a partir de la colaboración de diferentes agentes socioeducativos presentes y activos en la zona. En ambos casos, la implementación de los pilotajes ha requerido un trabajo de coordinación, llevado a cabo por el Centre Cruilla y Fundación IRES, respectivamente, que impulsan la gestión, la organización y la adaptación de las mesas de trabajo al contexto particular. Las mesas se rigen por un liderazgo compartido a partir de la igualdad entre los agentes participantes donde cada uno tiene un rol definido y donde la responsabilidad es también compartida.

Los actores manifiestan que este sistema de organización ha sido muy costoso en términos de tiempo y de trabajo de gestión, lo que ha significado en ambos casos alrededor de un año de un trabajo de construcción conjunta del proyecto piloto con los agentes participantes. En relación con este punto añaden que lo que podría ser considerado una demora, puede ser visto también como una fortaleza, ya que las bases de colaboración, los criterios y las condiciones son más sólidos y compartidos, aunque falta mejorar la corresponsabilidad y el compromiso constante por parte de los diferentes agentes implicados.

Se valora positivamente que el proceso de construcción de los pilotajes se haya basado en una metodología flexible y participativa, en la cual los diferentes agentes han sido invitados a construir conjuntamente la experiencia y a definir las formas de trabajo, consensuar los criterios de acción, generar consensos y documentos compartidos. Se constata que todo ello ha permitido avanzar en la planificación estratégica del proyecto.

Al mismo tiempo, los actores entrevistados coinciden que este proceso de aprendizaje mediante la acciónreflexión conjunta y compartida está promoviendo la creación un tejido de relaciones y sinergias y, por lo tanto, la proyección futura y la sostenibilidad en el tiempo de las experiencias. Por el momento, un primer resultado visible es la propia creación de estas redes y el establecimiento de criterios comunes para emprender la acción socioeducativa con la infancia y sus familias en los diferentes territorios. Cabrá esperar por lo menos un año o dos para observar los logros e incidencia de los pilotajes en la transformación del contexto.

\section{Discusión y conclusiones}

El programa CPI está concluyendo la fase de pilotaje de construcción de redes de acción socioeducativa en el territorio. Se trata de una aplicación del modelo de 
red centrada en la mejora de la inclusión socioeducativa de la infancia y adolescencia con mayor vulnerabilidad y menos recursos para acceder a oportunidades. Dada la temprana fase de desarrollo de las experiencias, solo podemos presentar, como primeras conclusiones, algunas reflexiones y aprendizajes en torno a los resultados «procesuales» de esta propuesta teórico-práctica de acción. En el caso de la ciudad de Barcelona se ponen de relieve tres grandes cuestiones alrededor de este emergente modelo de acción socioeducativa.

El proceso de construcción de redes se enfrenta a la dificultad que supone el cambio de paradigma en la acción social. Si bien los pilotajes suponen una experimentación de la evolución lógica de CPI, desde los planteamientos de acción integral, de calidad, eficiente y sostenible-planteamientos compartidos por todos los participantes en las experiencias- en la práctica, las culturas de trabajo basadas en la perspectiva uniprofesional pueden actuar como frenos del proceso. Sin embargo, las experiencias iniciadas en la ciudad de Barcelona han configurado ya equipos interdisciplinares con profesionales de diferentes servicios del territorio (educadores, trabajadores sociales, psicólogos, técnicos comunitarios y/o pediatras), lo que constituye un primer paso para empezar los procesos de acompañamiento socioeducativo a niños y jóvenes y a sus familias en situación de pobreza y vulnerabilidad social. Tenemos constancia desde la evaluación de otras iniciativas con trayectorias de más de una década de acción socioeducativa en red que esta forma de trabajo no solo optimiza los recursos, sino que también es una propuesta que da más oportunidades de inclusión social y educativa a los participantes, por ejemplo, en el ámbito del fracaso y absentismo escolar (Almirall et al., 2011; Civís \& Longás, 2015).

Si bien aludíamos a la necesidad de superar las diferencias marcadas por las distintas culturas profesionales y las limitaciones derivadas de las propias organizaciones no pensadas para trabajar en red, en todos los casos debe destacarse la buena acogida de la propuesta por parte de los profesionales de las diferentes entidades e instituciones implicadas, así como su interés por compartir, construir conjuntamente conocimiento y abrir ventanas a la innovación. Evaluaciones en torno a la metodología de trabajo en red en proyectos socioeducativos ponen de manifiesto el capital social añadido que aportan este tipo de experiencias a los profesionales (Almirall et al., 2011). En los pilotajes de CPI se confirma la importancia de respetar los tiempos y tener claro que el trabajo en red generará vínculos y nuevas incorporaciones durante el proceso de construcción. Así, a medida que avanzan la comunicación, el intercambio de información, el análisis conjunto de la realidad y el trabajo que supone la construcción y consolidación de la red, confluyen las perspectivas y visiones de sus miembros, generando conocimiento compartido y un estilo propio de trabajo.

Finalmente, también podemos concluir que el desarrollo y la implementación de protocolos comunes para regular los procedimientos concretos que deben seguirse en el acompañamiento de los niños y las niñas -evaluación compartida, acogida, derivaciones, coordinaciones, referentes,...- ayudan a reconocerse mutuamente, discutir y consensuar marcos de actuación, mejorar la comprensión del objeto de trabajo y optimizar el trabajo y los recursos. Si bien el avance en esta dirección está exigiendo encontrar vías para superar estructuras demasiado rígidas que no facilitan la colaboración intersectorial, se identifican progresos importantes de diálogo, confianza y logros en forma de acuerdos. De momento se optó por iniciar el trabajo en red con este esfuerzo concreto, válido para promover la relación entre los agentes del territorio por atender a una demanda (muy habitual en casi toda la ciudad) de coordinación de la acción que ya se realiza. Queda pendiente activar la otra finalidad de las redes: la gobernanza socioeducativa, más abstracta y de resultados a largo plazo, pero que constituye la verdadera esencia del trabajo en red a escala local.

\section{Referencias}

Almirall, R., Díaz-Gibson, J., Dot, M., Moya, J., Ramírez, L. \& Ubieto, J.R. (2011). Informe d'avaluació dels costos $i$ beneficis del model Interxarxes. Estudi valoratiu de l'experiència del programa Interxarxes (2000-2010). Barcelona: Ajuntament de Barcelona. Dte. d'HortaGuinardó. Divisió de Serveis Personals.

Athanasoglou, S. \& Dijkstra, L. (2014). The Europe 2020 Regional Index. Science and Policy Report. Luxembourg: Publications Office of the European Union.

Ballester, L. (2014). Redes sociales y escuela. En M.X. March Cerdà \& C. Orte Socias (Coords.), La pedagogía social y la escuela. Los retos socioeducativos de la institución escolar en el siglo XXI (pp.185-206). Barcelona: Octaedro.

Boada, C., Herrera, D., Mas, E., Miñarro, E., Olivella, M. \& Riudor, X. (dir.). (2011). Informe sobre el risc de fracàs escolar a Catalunya. Barcelona: Generalitat de Catalunya. Consell de Treball, Econòmic i Social de Catalunya, Col-lecció Estudis i Informes, (26).

Boadas, B. \& Ricciardelli, O. (2012). CaixaProinfancia. Informe estudio del mapeo. 2012. Zona de Barcelona. Barcelona: Grupo de investigación PSITIC (URL). [Informe de investigación sobre el programa CaixaProinfancia no publicado].

Calero, J., Chois, K. \& Waisgrais, S. (2010). Determinantes del riesgo de fracaso escolar en España: una aproximación a través de un análisis logístico multinivel aplicado a PISA-2006. Revista de Educación, número extraordinario 2010, 225-256. doi:10.4438/ 1988-592X-0034-8082-RE

Civís, M. \& Longás, J. (2015). La colaboración interinstitucional como respuesta al desafío de la inclusión socioeducativa. Análisis de 4 experiencias de trabajo en red a niel local en Cataluña. Educación XXI, 18(1), 213-236. doi:10.5944/educXX1.18.1.12318

Civís, M. \& Riera, J. (2007). La nueva pedagogía comunitaria. Un marco renovado para la acción sociopedagógica interprofesional. Valencia: Nau llibres. 
Coller, X. (2000). Estudio de casos. Madrid: Centro de Investigaciones Sociológicas. Cuadernos Metodológicos, 30.

Comisión Europea. (2010). Europa 2020. Una estrategia para un crecimiento inteligente, sostenible e integrador. Bruselas: Comisión Europea.

Departament d'Estadística. Ajuntament de Barcelona (2012a). Pobresa i exclusió social. 2011. Taxa de risc a la pobresa segons edat. Total població. Enquesta de condicions de vida i hàbits de la població. Idescat i IERMB. Recuperado de 03/02/2014, http://www.bcn.cat/estadistica/catala/dades/tvida/ecvhp/a2011/e73.htm

Departament d'Estadística. Ajuntament de Barcelona (2012b). Pobresa i exclusió social. 2011. Població en risc de pobresa o exclusió. Total població. Enquesta de condicions de vida $i$ hàbits de la població. Idescat i IERMB. Recuperado de 03/02/2014, http://www.bcn.cat/estadistica/catala/dades/tvida/ecvhp/a2011/e712.htm

Díaz-Gibson, J., Civís, M., Longás, J. \& López, A. (2010). The Study of Educative Network organization in the City of Barcelona, Spain: The Nou Barris District. International Journal of Knowledge Society Research, 1(2), 26-37. doi:10.4018/jksr.2010040103

Díaz-Gibson, J. \& Civís, M. (2011). Redes Socioeducativas promotoras de capital social en la comunidad: un marco teórico de referencia. Cultura y Educación, 23(3), 415-429. doi: 10.1174/113564011797330270

Díaz-Gibson, J., Civís, M. \& Longás, J. (2013). La gobernanza de redes socioeducativas: claves para una gestión exitosa. Teoría de la Educación, 25(2), 213-230.

Eurostat. (2015). People at risk of poverty or social exclusion by age and sex (AROPE). 2013. [ilc_peps01, última modificación 16/04/2015]. Recuperado de 21/04/2015, http://ec.europa.eu/eurostat/data/database

Garcia Alegre, E. (2013). Èxit educatiu i pobresa infantil a Catalunya. Reptes de les polítiques socioeducatives. En M. Martínez Martín \& B. Albaigés Blasi (Dirs.), L'estat de l'educació a Catalunya. Anuari 2013, (pp. 415-424). Barcelona: Fundació Jaume Bofill.

Gil-Flores, J. (2011). Estatus socioeconómico de las familias y resultados educativos logrados por el alumnado. Cultura y Educación, 23(1), 141-154. doi: $10.1174 / 113564011794728597$

González-Bueno, G., Bello, A. \& Arias, M. (2012). La infancia en España 2012-2013. El impacto de la crisis en los niños. Madrid: UNICEF. Recuperado de 10/07/2013, http://www.unicef.es/sites/www.unicef. es/files/Infancia_2012_2013_final.pdf

González-Bueno, G. \& Bello, A. (2014). La infancia en España 2014. El valor de los niños. Hacia un Pacto de Estado por la Infancia. Madrid: UNICEF Comité Español. Recuperado de 19/10/2014, http://www.unicef. es/sites/www.unicef.es/files/infancia-espana/unicef_ informe_la_infancia_en_espana_2014.pdf

Grupo de investigación PSITIC. (2011). Programa CaixaProinfancia: modelo de promoción y desarrollo integral de la infancia en situación de pobreza y vulnerabilidad social. Barcelona: Obra Social «la Caixa».

Grupo de investigación PSITIC (2013a). Programa CaixaProinfancia: modelo de promoción y desarrollo integral de la infancia en situación de pobreza y vulnerabilidad social. ( $2^{a}$ edición). Barcelona: Obra Social «la Caixa».

Grupo de investigación PSITIC (2013b). Modelo de acción social Programa CaixaProinfancia. Barcelona: Obra Social «la Caixa».

Grupo de investigación PSITIC (2013c). Guía del Refuerzo Educativo Programa CaixaProinfancia. Barcelona: Obra Social «la Caixa».

Grupo de investigación PSITIC (2013d). Guía de Atención Psicoterapéutica Programa CaixaProinfancia. Barcelona: Obra Social «la Caixa».

Idescat. Institut d'Estadística de Catalunya. (2012). Territori. Banc d'estadístiques de municipis i comarques. Estructura de la població. Padró municipal d'habitants. Sexe i Edat. Sexe i Edat Any a Any. 2012. Recuperado de 03/02/2014, http://www.idescat.cat/territ/ BasicTerr?TC=9

Idescat. Institut d'Estadística de Catalunya. (2014). Població en risc de pobresa o exclusió. Menors 16 anys. Barcelona. Enquesta de Condicions de Vida i Hàbits de la Població, 2011 (ECVHP, 2011). Barcelona: Idescat y IERMB. [Datos disponibles el 23/07/2014, proporcionados por Idescat a partir de demanda específica de explotación de la ECVHP 2011].

INE. Instituto Nacional de Estadística. (2012). Encuesta de Condiciones de Vida Año 2011. Transmisión intergeneracional de la pobreza y el bienestar. Nota de prensa, 08-11-2012. Madrid: INE. Recuperado de 28/06/2013, www.ine.es

INE. Instituto Nacional de Estadística. (2014a). Tasa de riesgo de pobreza o exclusión social (estrategia Europa 2020) por tipo de hogar. Encuesta de Condiciones de Vida. Series Base 2013. Resultados nacionales, publicado 27 Mayo 2014. Madrid: INE. Recuperado de 27/04/2015, www.ine.es

INE. Instituto Nacional de Estadística. (2014b). Tasa de riesgo de pobreza o exclusión social (estrategia Europa 2020) por nivel de formación alcanzado (personas de 16 y más años). Encuesta de Condiciones de Vida. Series Base 2013. Resultados nacionales, publicado 27 Mayo 2014. Madrid: INE. Recuperado de 27/04/2015, www. ine.es

INE. Instituto Nacional de Estadística. (2014c). Tasa de riesgo de pobreza o exclusión social (estrategia Europa 2020) por nacionalidad (personas de 16 y más años). Encuesta de Condiciones de Vida. Series Base 2013. Resultados nacionales, publicado 27 Mayo 2014. Madrid: INE. Recuperado de 27/04/2015, www.ine. es

INE. Instituto Nacional de Estadística. (2015). Tasa de paro por nivel de formación alcanzado, sexo y grupo de edad. Encuesta de Población Activa. Resultados nacionales, $1 r$ trimestre 2015, publicados 23 de Abril de 2015. Madrid: INE. Recuperado de 27/04/2015, www.ine. es

Laguna, E. \& Riera, J. (1998). La interdisciplinarietat. Aloma: Revista de Psicologia, Ciències de l'Educació i de l'Esport Blanquerna, 2, 15-24.

Longás, J. \& Civís, M. (coords.) (2010). Estudi de les 
xarxes socioeducatives de Nou Barris. Fortaleses i oportunitats. Informe de recerca. Barcelona: Universitat Ramon Llull.

Marchesi, A. \& Hernández Gil, C. (2003). El fracaso escolar. Una perspectiva internacional. Madrid: Alianza Editorial.

MECD. Ministerio de Educación, Cultura y Deporte. (2013). PISA 2012: Programa para la Evaluación Internacional de los Alumnos. Informe Español. Volumen 1: Resultados y Contexto. OCDE. Madrid: Ministerio de Educación, Cultura y Deporte. Recuperado de 20/04/2015,

http://www.mecd.gob.es/dctm/inee/internacional/ pisa2012/pisa2012lineavolumeni.pdf?documentId= 0901e72b81786310

Riera, J. (1997). La interprofessionalitat. Aloma: Revista de Psicologia, Ciències de l'Educació i de l'Esport Blanquerna, 1, 15-22.

Riera, J. (2012). L'educació és una de les estratègies principals perquè la pobresa deixi de ser hereditària. Entrevista al Dr. Jordi Riera. Butlletí Blanquerna, març 2012, (163). Barcelona: Gabinet de Comunicació Blanquerna URL. Recuperado de 10/09/2013, http:// premsa.blanquerna.edu/butlleti/Entrevista.aspx? NumButlleti $=163$

Riera, J. \& Cussó, I. (2013). Aproximació multidimensional a la pobresa infantil. Vers la creació d'estratègies d'acció integrals i en xarxa de corresponsabilitats socioeducatives, Educació Social Revista d'Intervenció Socioeducativa, 52, 109-129.
Sarasa, S. \& Sales, A. (2009). Itineraris i Factors d'Exclusió Social. Barcelona: Ajuntament de Barcelona. Síndica de Greuges de Barcelona. Recuperado de 20/04/2015, http://www.sindicadegreugesbcn.cat/pdf/monografics/itineraris.es.pdf

Acció socioeducativa en xarxa amb infants i adolescents en situació de pobresa: anàlisi del programa CaixaProinfància a Barcelona

Resum. L'article presenta com a estudi de cas l'aplicació del programa CaixaProinfància a Barcelona impulsat per l'Obra Social de la Fundació Bancària Caixa d'Estalvis i Pensions de Barcelona, «la Caixa». S'explica el marc teòric del programa $i$ s'analitza la seva incidència i evolució a la ciutat. Dels resultats de la investigació, cal destacar les raons que han justificat el progressiu canvi cap a un plantejament exigent de treball socioeducatiu en xarxa. Al seu torn, a partir de les noves experiències, s'exposen els encerts, les expectatives i limitacions trobades fins al moment en la implementació d'aquest model emergent d'acció socioeducativa. Els resultats apunten cap a una acció més sostenible i eficaç en l'atenció integral de la infància gràcies al treball col-laboratiu en el territori.

Paraules clau: pobresa infantil; acció socioeducativa; inclusió social; treball en xarxa

El proyecto ha sido financiado por la Obra Social de la Fundación Bancaria Caixa d'Estalvis i Pensions de Barcelona, "La Caixa"; Irene Cussó-Parcerisas cuenta con el apoyo del Programa de FPU del MECD 
